\title{
Chronic infection by nontypeable Haemophilus influenzae fuels airway inflammation
}

\author{
Fabio Saliu (10 ${ }^{1,2,3}$, Giulia Rizzo ${ }^{2,3}$, Alessandra Bragonzi ${ }^{3}$, Lisa Cariani ${ }^{4}$, \\ Daniela M. Cirillo $0^{1}$, Carla Colombo ${ }^{5}$, Valeria Daccò ${ }^{5}$, Daniela Girelli ${ }^{4}$, \\ Sara Rizzetto ${ }^{4}$, Barbara Sipione ${ }^{3}$, Cristina Cigana $\mathbb{1}^{3}$ and Nicola I. Lorè $\mathbb{1}^{1,2,3}$
}

Affiliations: 'IRCCS San Raffaele Scientific Institute, Division of Immunology, Transplantation, and Infectious Diseases, Emerging bacterial pathogens Unit, Milan, Italy. ${ }^{2}$ Università Vita-Salute San Raffaele, Milan, Italy. ${ }^{3}$ IRCCS San Raffaele Scientific Institute, Division of Immunology, Transplantation, and Infectious Diseases, Infections and cystic fibrosis unit, Milan, Italy. ${ }^{4}$ Cystic Fibrosis Microbiology Laboratory, Fondazione IRCCS Ca Granda, Milan, Italy. ${ }^{5}$ Cystic Fibrosis Regional Reference Center, Fondazione IRCCS Ca' Granda, Ospedale Maggiore Policlinico, University of Milan, Milan, Italy.

Correspondence: Nicola I. Lorè IRCCS Ospedale San Raffaele, Via Olgettina n. 60, 20132 Milano, Italy. E-mail: lore.nicolaivanahsr.it

ABSTRACT Nontypeable Haemophilus influenzae (NTHi) is commonly isolated from airways of patients suffering from chronic respiratory diseases, such as COPD or cystic fibrosis (CF). However, to what extent NTHi long-term infection contributes to the lung inflammatory burden during chronic airway disease is still controversial.

Here, we exploited human respiratory samples from a small cohort of CF patients and found that patients chronically infected with NTHi had significantly higher levels of interleukin (IL)-8 and CXCL1 than those who were not infected. To better define the impact of chronic NTHi infection in fuelling inflammatory response in chronic lung diseases, we developed a new mouse model using both laboratory and clinical strains. Chronic NTHi infection was associated with chronic inflammation of the lung, characterised by recruitment of neutrophils and cytokine release keratinocyte-derived chemokine (KC), macrophage inflammatory protein 2 (MIP-2), granulocyte colony-stimulating factor (G-CFS), IL-6, IL-17A and IL-17F) at 2 and 14 days post-infection. An increased burden of T-cell-mediated response $\left(\mathrm{CD}^{+}{ }^{+}\right.$and $\gamma \delta$ cells) and higher levels of pro-matrix metalloproteinase 9 (pro-MMP9), known to be associated with tissue remodelling, were observed at 14 days post-infection. Of note we found that both $\mathrm{CD} 4^{+} \mathrm{IL}-17^{+}$cells and levels of IL-17 cytokines were enriched in mice at advanced stages of NTHi chronic infection. Moreover, by immunohistochemistry we found $\mathrm{CD}^{+}, \mathrm{B} 220^{+}$and CXCL- $13^{+}$cells localised in bronchusassociated lymphoid tissue-like structures at day 14.

Our results demonstrate that chronic NTHi infection exerts a pro-inflammatory activity in the human and murine lung and could therefore contribute to the exaggerated burden of lung inflammation in patients at risk.

@ERSpublications

The pathological impact of long-term infection by nontypeable Haemophilus influenzae (NTHi) is still debated. Chronic NTHi infection fuels lung inflammation in human samples and in a new mouse model of bacterial long-term persistence. https://bit.ly/3lvyvge

Cite this article as: Saliu F, Rizzo G, Bragonzi A, et al. Chronic infection by nontypeable Haemophilus influenzae fuels airway inflammation. ERJ Open Res 2021; 7: 00614-2020 [https:// doi.org/10.1183/23120541.00614-2020].

This article has supplementary material available from openres.ersjournals.com

Received: 10 Sept 2020 | Accepted after revision: 6 Nov 2020

Copyright $\odot$ ERS 2021. This article is open access and distributed under the terms of the Creative Commons Attribution Non-Commercial Licence 4.0. 


\section{Introduction}

Recurrent or chronic infections by several opportunistic pathogens are common in patients affected by different chronic respiratory diseases, including COPD and cystic fibrosis (CF) [1-3]. Infections by nontypeable Haemophilus influenzae (NTHi), a non-capsulated Gram-negative bacterium, are associated with a worse clinical prognosis in several chronic respiratory diseases, such as COPD [4, 5], while its pathogenic potential in CF disease is still controversial [1].

Vicious cycles of chronic inflammation and infections, characterised by exaggerated and continuous recruitment of neutrophils in the airways via powerful inflammatory signals, such as interleukin (IL)- 8 or IL-6 $[6,7]$, have often been associated with immunopathology and decline in pulmonary function among patients suffering from chronic respiratory diseases $[8,9]$. In this context, the IL-17-mediated host response is an emerging immunological determinant influencing pulmonary defence and function in several pathologies, including COPD or CF lung disease $[6,10,11]$. For example, IL-17-mediated immunity has been shown to modulate host defence against opportunistic pathogens (e.g. Pseudomonas aeruginosa), as well as the exaggerated neutrophil recruitment generated during bacterial chronic infection [12-14].

It has been reported that the presence of NTHi can be associated with acute exacerbation episodes in CF [15]. Similar observations have been made in COPD patients, although in this case the NTHi infection was also associated with a worse clinical prognosis $[16,17]$. However, there are no observations on the effect of chronic NTHi infection in the progression of CF chronic airway disease, due to the presence of several clinical and microbiological cofounding factors, such as concomitant infections [15, 18] and lack of available human cohorts. Nonetheless, it has been observed that patients with COPD and chronically colonised with Haemophilus influenzae during stable disease phase display sustained airway inflammation $[18,19]$. So far, to what extent long-term NTHi infection contributes to the lung inflammatory burden during chronic airway disease is controversial, and animal models could be instrumental to clarify its role in fuelling inflammation. Previous experimental studies on NTHi were mainly focussed on the acute virulence of genotypically and phenotypically different bacterial isolates [20-23] rather than on the pathogenic potential of chronic NTHi infection in the airways.

However, at the time of this writing, no experimental studies have developed a model of long-term NTHi infection in murine airways that strictly mimics human infectious processes. Exaggerated inflammation, immunopathology, pulmonary damage and remodelling are difficult to reproduce in mice using the available NTHi transient infection models. As a consequence, the cascade of events mediated by chronic NTHi infection has been difficult to address.

Here, to validate our hypothesis that chronic NTHi infection contributes to fuelling the inflammatory burden in CF lung, we measured the concentrations of a few pro-inflammatory cytokines, related to neutrophil recruitment, in respiratory samples from CF patients infected by NTHi alone or not infected by any CF opportunistic pathogens. In addition, NTHi laboratory and clinical strains were used to test their pathogenic potential 1) in a new mouse model for chronic infection mirroring clinical chronic NTHi lung infection and 2) in in vitro host-pathogen experiments. Overall, our data demonstrate that chronic NTHi infection, independent of NTHi phenotypic diversity, may contribute to fuelling the exaggerated inflammatory burden in the airways of patients suffering from chronic respiratory disease.

\section{Methods}

\section{Ethical committee}

Animal studies were performed according to the protocols set out by the Italian Ministry of Health guidelines for the use and care of experimental animals (IACUC protocols \#920) and approved by the San Raffaele Scientific Institute (Milan, Italy), Institutional Animal Care and Use Committee (IACUC). Human samples and the bacteria from CF patients were collected at the Cystic Fibrosis Center of Milan, Italy. The study was approved by the Ethical Committees of San Raffaele Scientific Institute and of Fondazione IRCCS Ca' Granda, Ospedale Maggiore Policlinico, Milan, Italy. Written informed consent was obtained from enrolled patients, or their parents, according to the Ethical Committee rules, in accordance with the laws of the Italian Ministero della Salute (approvals \#1874/12 and 1084/14).

\section{Human respiratory samples and lung function}

Nasopharyngeal aspirates from 19 clinically stable CF patients with variable genotypes were collected from the Regional CF Center at Milan's Ospedale Maggiore Policlinico during routine care visits as described in supplementary methods 1. Seven patients (five females, 13-20 years old) were negative for CF opportunistic pathogens; 12 patients (five females, 4-9 years old) were infected with NTHi but negative for other opportunistic pathogens. Further details are provided in the supplementary methods and supplementary table S1. 


\section{Bacterial strains}

ATCC 49766 is a reference NTHi laboratory strain. The eight NTHi clinical strains tested in in vivo or in vitro were isolated from CF patients and identified by CF Microbiology Laboratory, IRCCS Ca' Granda, Ospedale Maggiore Policlinico. Further details are provided in supplementary table S2.

\section{Mouse model of chronic NTHi lung infection}

Persistent airway infection was obtained by intratracheal injection of agar beads containing $\sim 1 \times 10^{7} \mathrm{CFU}$ of NTHi (ATCC 49766 or NTHi 50, a CF clinical strain) or empty beads (as sham control) in 20- to 22-g C57BL6/NCrl male mice (8-10 weeks, Charles River). Agar beads were produced by refining a previously described protocol $[13,14,24,25]$ as described in supplementary methods. Sham-infected mice were used as control mice. Animals were euthanised after 2, 7 and 14 days by $\mathrm{CO}_{2}$ administration, and lungs were recovered. Sample processing was performed as described in supplementary methods.

\section{Flow cytometry and intracellular cytokines staining}

After euthanasia, lungs were harvested and mechanically dissociated in GentleMACS C tubes (Miltenyi Biotec, Bergisch Gladbach, Germany) using GentleMACS dissociators, followed by straining through a $70-\mu \mathrm{m}$ filter. Antibody staining was performed as previously described [24, 26]. Briefly, lung cell suspensions $\left(1.5 \times 10^{6}\right.$ cells $)$ were incubated with blocking buffer $(5 \%$ rat serum and $95 \%$ culture supernatant of $24 \mathrm{G} 2$ anti-FcR mAb-producing hybridoma cells) for $10 \mathrm{~min}$ at $4^{\circ} \mathrm{C}$. Then, cells were stained for $20 \mathrm{~min}$ at $4^{\circ} \mathrm{C}$ in the dark with different combinations of antibodies (supplementary tables S3, S4 and S5). For intracellular cytokine staining, $1-3 \times 10^{6}$ cells were stimulated with phosphomolybdic acid (PMA) $\left(3 \mathrm{ng} \cdot \mathrm{mL}^{-1}\right)$ and ionomycin $\left(1 \mu \mathrm{g} \cdot \mathrm{mL}^{-1}\right)$ for $4 \mathrm{~h}$ at $37^{\circ} \mathrm{C}$ (the last $2 \mathrm{~h}$ with Brefeldin A $5 \mu \mathrm{g} \cdot \mathrm{mL}^{-1}$ ). Cells were surface stained, fixed (paraformaldehyde $2 \%$ for $10 \mathrm{~min}$ ), permeabilised ( $2 \%$ fetal bovine serum (FBS), $0.2 \% \mathrm{NaN}_{3}, 2 \%$ rat serum, $0.5 \%$ saponin in Dulbecco's phosphate-buffered saline (DPBS)) and then stained for intracellular cytokines for $30 \mathrm{~min}$ at room temperature in darkness. Acquisition and analyses were performed using a fluorescence-activated cell sorting FACS Canto cytometer (BD Biosciences, San Jose, CA, USA) and FlowJo Software (Tree Star, Ashland, OR, USA), as previously described [14, 26]. Further details are provided in the supplementary material.

\section{Histological analysis}

Lungs were removed and fixed in $4 \%$ paraformaldehyde. After paraffin embedding, consecutive $5-\mu \mathrm{m}$ sections from the middle of the five lung lobes were used for histological and immunohistochemical examination. Sections were stained with haematoxylin and eosin (H\&E) or immunostained as previously described with anti-CD3, anti-B220, anti-CXCL-13, anti-F4/80 antibodies [27]. A description of the primary antibodies, together with dilutions and unmasking techniques, is provided in supplementary table S6. Images were acquired using the Aperio CS2 scanner (Leica Biosystems, Nussloch, Germany) and analysed with the Aperio ImageScope software (Leica Biosystems).

\section{Morphometric analysis}

A quantitative analysis was performed on tissue slides using the Aperio ImageScope software. Lymphoid aggregates were defined as cell clusters positive for the CD3 molecule and were quantified as number of clusters per square centimetre of lung.

\section{ELISA}

Concentration of cytokines/chemokines in lung homogenates was assessed by ELISA using DuoSet kits (R\&D Systems, Minneapolis, MN, USA) for growth-regulated $\alpha$ protein/keratinocyte-derived chemokine (CXCL1/KC), C-X-C motif chemokine 2/macrophage inflammatory protein 2 (CXCL2/MIP-2), granulocyte colony-stimulating factor (G-CSF), IL-6, IL-17A, IL-17F and pro-matrix metalloproteinase 9 (Pro-MMP9) according to the manufacturer's instructions.

\section{NTHi infection in A549 cell line}

Human alveolar epithelial A549 cells were cultured and challenged with NTHi strains as described in the supplementary methods. Cell viability was assessed by measuring the lactate dehydrogenase released in the supernatant with CytoTox 96 Non-Radioactive Cytotoxicity Assay (Promega, Madison, WI, USA) following the manufacturer's instructions.

\section{Statistical analysis}

Statistical analysis was performed with GraphPad Prism (GraphPad Software, San Diego, California, USA) for statistical computing. Data at a specific time-point were compared through a nonparametric two/ one-tailed Mann-Whitney test. Incidences of mortality and chronic infection were compared using Fisher's exact test. Statistical analyses were considered significant at $\mathrm{p}<0.05$. 


\section{Results}

Impact of NTHi colonisation on chemokines associated with neutrophil recruitment in CF lung

We evaluated the levels of CF pro-inflammatory cytokines (IL-8 and CXCL1) in respiratory samples of 19 clinically stable CF patients with NTHi infection or not infected by NTHi (see Material and methods and supplementary table S1). We found that CF patients infected with NTHi had significantly higher levels of both IL-8 $(\mathrm{p}<0.05)$ and CXCL1 $(\mathrm{p}<0.05)$ than those uninfected (figure 1a and $b)$. These data suggest that chronic NTHi infection may contribute to increase the levels of chemokines recruiting neutrophils in CF lung.

\section{Development of a new murine model to mirror chronic NTHi infection as observed in human pathology}

To investigate whether NTHi infection can induce a sustained inflammatory response, we developed a new murine model that mirrors chronic NTHi infection as observed in human CF pathology.

We adapted a murine model, previously developed for chronic bacterial lung infections [13, 14], to study chronic NTHi infection in lower-middle airways. We developed chronic airways infection with the NTHi reference strain 49766 over 14 days with bacterial loads of $\approx 10^{6} \mathrm{CFU} \cdot \mathrm{lun}^{-1}$ (figure 2a), resulting in a high incidence of chronic infection (80\%) at 14 days post-infection (figure $2 \mathrm{~b}$ ). No CFUs were detected in the spleens of infected mice, indicating that NTHi was confined to the lung compartment and did not spread systemically.

Focussing on the early (2 days) and advanced (14 days) stages of infection, we evaluated histopathology and the levels of pro-inflammatory cytokines/chemokines related to neutrophil recruitment into the lungs. $\mathrm{H} \& \mathrm{E}$ staining on day 2 and 14 days post-infection localised the agar beads to bronchial lumens. In term of inflammation, the early phase was mainly characterised by the acute neutrophil and macrophage recruitment (figure 2c), while the chronic NTHi infection was featured also by adaptive immune response including the formation of organised macrostructures of lymphocytes (figure $2 \mathrm{~d}$ ) similar to those observed for chronic $P$. aeruginosa infection $[13,14,28]$.

The levels of KC, MIP-2 (both murine functional homologues of human IL-8 [29]) and IL-6 were significantly increased in comparison to sham-infected (control) mice at 2 or 14 days post-infection (KC/ MIP-2/IL-6: control versus 2 days $\mathrm{p}<0.05$, control versus 14 days $\mathrm{p}<0.05)$, reflecting the higher recruitment of innate immune cells described in the histopathological analysis (figure $2 \mathrm{e}, \mathrm{f}$ and $\mathrm{h}$ ). Moreover, the levels of IL-17A, IL-17F and G-CSF were also significantly higher (IL-17A/IL-17F/G-CSF: control versus 2 days $\mathrm{p}<0.05$, control versus 14 days $\mathrm{p}<0.01$ ) than those found in sham-infected mice at 2 and 14 days (figure $2 \mathrm{~g}$, $\mathrm{i}$ and 1). Of note, IL-17A levels increased by day 2 and remained high over the course of NTHi infection, suggesting the involvement of type 17 immunity. Overall, our new murine model can mirror the acute/ early and advanced stages of chronic lung infection observed during the course of human airway diseases.

\section{Chronic NTHi infection in the mouse lung promotes sustained type 17 immunity and remodelling} processes

In order to dissect the lung infiltrating cells, fluorescence-activated cell sorting (FACS) analysis was performed. The number of infiltrating leukocytes was significantly higher in samples recovered from
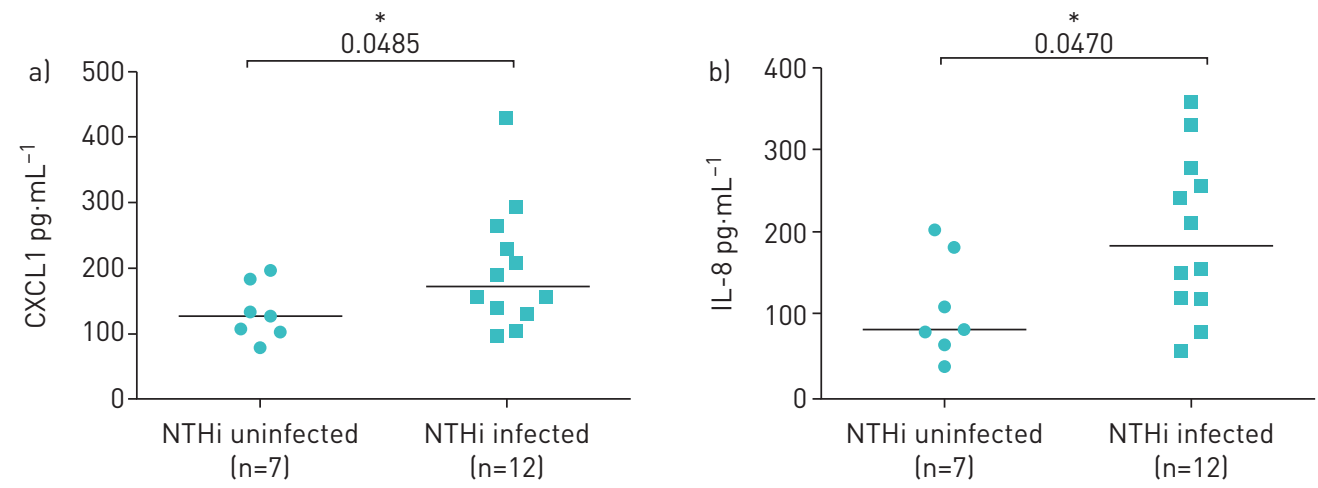

FIGURE 1 Contribution of nontypeable Haemophilus influenzae (NTHi) colonisation to lung inflammatory burden in cystic fibrosis (CF) patients. Levels of proinflammatory chemokines a) CXCL1 and b) interleukin (IL)-8 were evaluated by ELISA in respiratory samples from a retrospective study in CF patients infected exclusively with NTHi $(n=12)$ or uninfected $(n=7)$ by any recognised CF pathogen. *: $p<0.05$ compared with control (Mann-Whitney $t$-test). 
a)

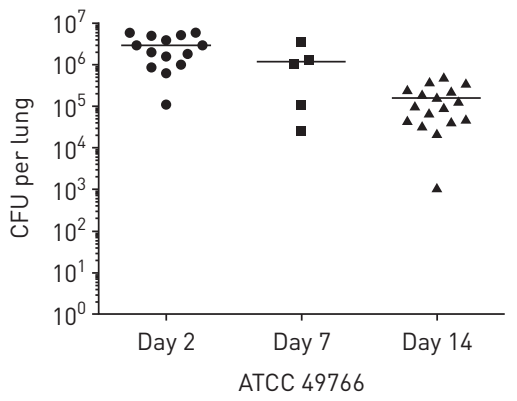

b)

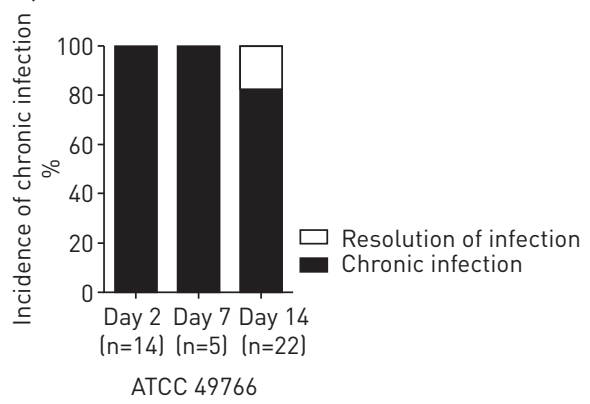

c)

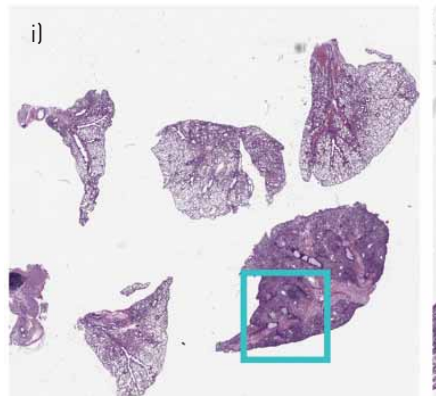

d)

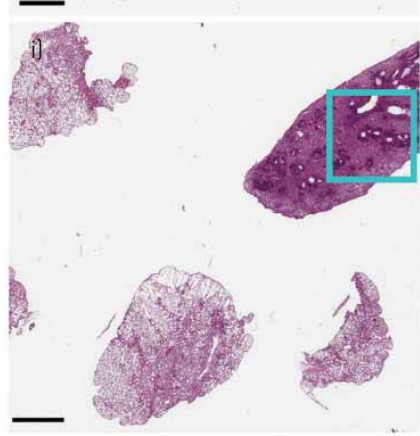

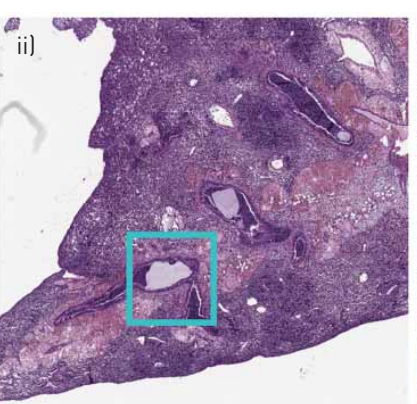

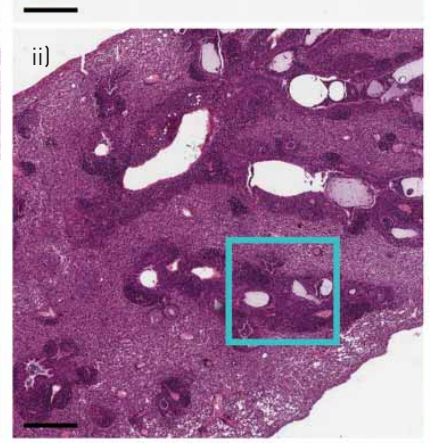

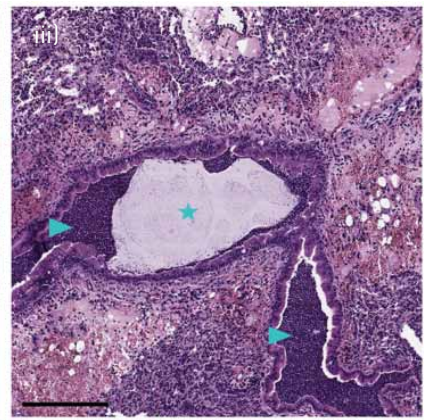
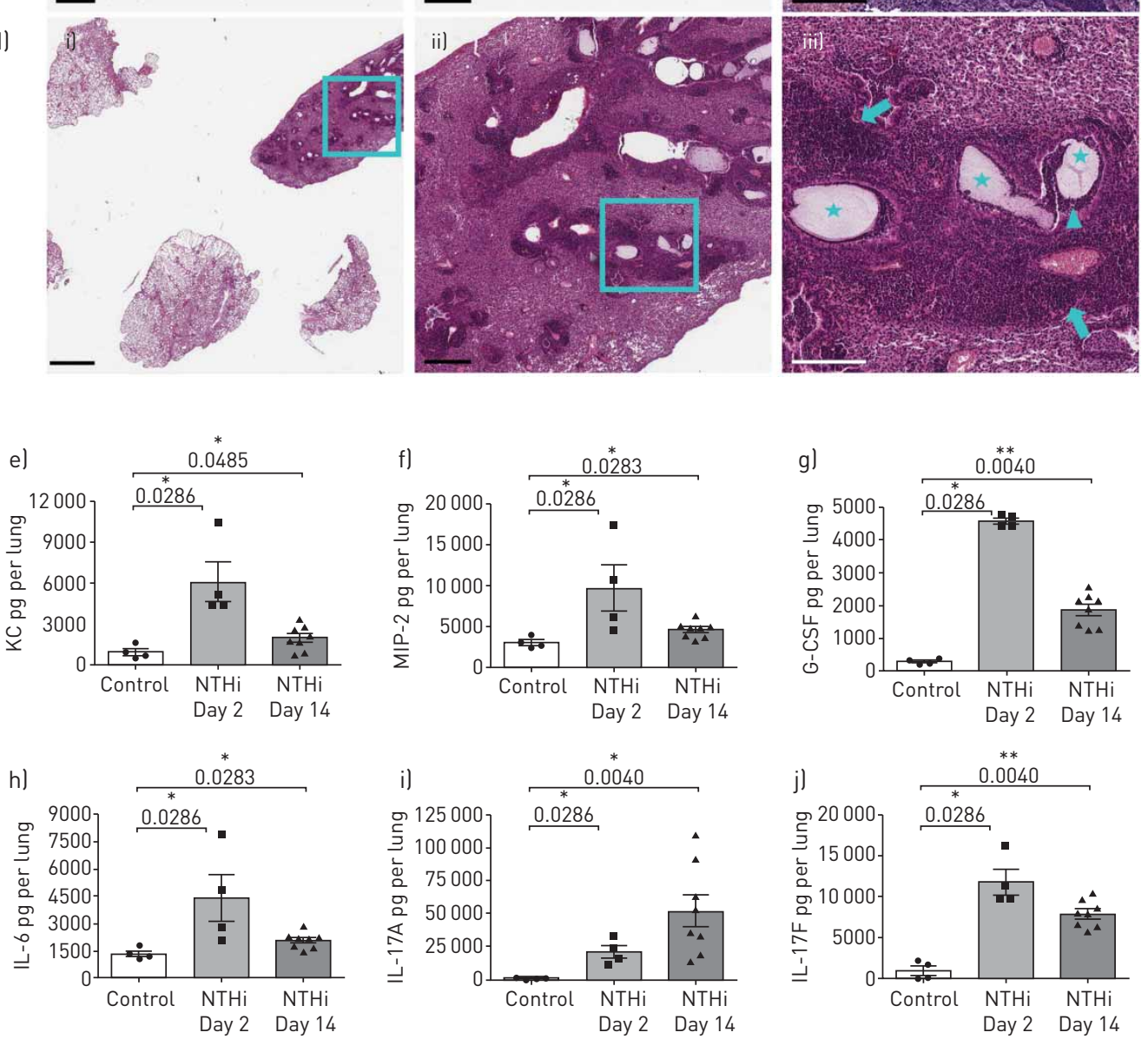

FIGURE 2 Bacterial burdens, histopathology and pro-inflammatory cytokines during nontypeable Haemophilus influenzae (NTHi) infection in murine airways. C57BL/6N mice were infected intratracheally with NTHi-embedded agar beads. Bacterial loads in the lung (a) and the incidence of chronic infection (b) were measured at 2, 7 and 14 days post-infection. Data are pooled from at least three independent experiments, $\mathrm{n}=14$ (day 2), $\mathrm{n}=5$ (day 7) and $\mathrm{n}=22$ (day 14). Haematoxylin and eosin staining of lung tissue sections was 
performed at 2 days (c) and 14 days (d) post-infection. Stars indicate the presence of agar beads in bronchial lumens, arrowheads indicate the presence of neutrophils and macrophages in bronchial lumens, and arrows indicate the presence of lymphocyte aggregates. Representative images are shown from the lower (i) to the higher (iii) magnification. i) scale bar $=2 \mathrm{~mm}$; ii) scale bar $=500 \mu \mathrm{m}$; iii) scale bar $=200 \mu \mathrm{m}$. keratinocyte-derived chemokine (KC) (e), MIP-2 (f), granulocyte colony-stimulating factor (G-CSF) (g), interleukin (IL)-6 (h), IL-17A (i) and IL-17F (j) concentration in the murine lungs was assessed by ELISA at both 2 and 14 days post-infection. ${ }^{*}$ : $p<0.05$; $^{* *}$ : $<<0.01$; and ${ }^{* * *}$ : $p<0.001$ compared with control (Mann-Whitney $t$-test).

infected mice at both day $2(\mathrm{p}<0.001)$ and $14(\mathrm{p}<0.001)$ post-infection when compared to control mice (figure 3a). Neutrophils and monocytes were recruited in infected lungs at both time points (figure $3 \mathrm{~b}$ and $\mathrm{c}$ ), while alveolar macrophages and dendritic cells were increased only at 14 days $(\mathrm{p}<0.001)$ post-infection (figure $3 \mathrm{~d}$ and e). On the other hand, neutrophil and monocyte counts decreased on day 14 post-infection, although they remained higher than in control mice. Additionally, T-cells were significantly $(\mathrm{p}<0.05)$ more represented among infected mice compared to control ones (figure 3f). While both $\gamma \delta^{+}$and $\mathrm{CD} 4^{+} \mathrm{T}$-cell subsets were significantly enriched both at day 2 and day 14 post-infection (figure $3 \mathrm{~g}$ and $\mathrm{h}$ ), $\mathrm{CD} 8^{+} \mathrm{T}$-cell subsets only increased at day 14 (figure $3 \mathrm{i}$ ).

We also found a selective enrichment of $\mathrm{CD} 4^{+} \mathrm{IL}-17 \mathrm{~A}^{+} \mathrm{T}$-cells $(\mathrm{p}<0.05)$, but not of $\mathrm{CD} 4^{+} \mathrm{IL}-4^{+}, \mathrm{CD} 4^{+}$ IFN- $\gamma^{+}$or $\mathrm{CD}^{+}$IL- $13^{+}$T-cells (figure $3 \mathrm{j}$ ). We quantified the levels of pro-MMP9 as a marker associated with tissue remodelling processes [30]. Levels of pro-MMP-9 were significantly upregulated $(p<0.05)$ during chronic NTHi infection in the lung (figure 3k), confirming that chronic NTHi infection is associated with sustained tissue remodelling processes. Thus, all together these data indicate that chronic infection by NTHi in the lower airways induces local type 17 immune manifestations and sustained tissue remodelling processes.

\section{Pathogenicity of a CF clinical isolate during chronic NTHi infection in murine airways}

We tested whether a CF NTHi isolate displayed a worse pathogenic potential in comparison to the reference laboratory strain during chronic lung infection. We exploited our new model of chronic NTHi infection in order to compare bacterial burdens and host response between the reference laboratory strain and a CF isolate, named "NTHi50". As shown in figure 4a and b, NTHi50 did not differ from the 49766 reference strain in terms of bacterial burden and incidence of chronic infection in C57BL/6N mice 14 days post-challenge. We then investigated whether the inflammatory response in terms of quality and burden could be different during chronic infection by those NTHi strains. We evaluated both histological lesions by the analysis of lung tissue slices stained with H\&E (figure 4c-e) and lung infiltrating cells by FACS analysis in the lung. Both analyses revealed that NTHi50 induced a host response similar to that due to the 49766 strain. As a matter of fact, the sustained immune response was characterised by T-cells, comprising both $\gamma \delta^{+}$and $\mathrm{CD}^{+}$subsets, and associated with sustained neutrophil recruitment (figure $4 \mathrm{f}-\mathrm{m})$. In addition, by immunohistochemistry, we found that $\mathrm{CD}^{+}$cells were localised in lymphocyte aggregates (figure 5 and supplementary figure S2) at the advanced stage of chronic infection with both strains. Moreover, we determined that $\mathrm{CD}^{+}$aggregates were characterised by the strong co-localisation of B220 and CXCL13-expressing cells, demonstrating the presence of bronchus-associated lymphoid tissue (BALT)-like structures at day 14.

To test whether the bacterial pathogenic contribution differs among CF NTHi isolates, we infected the A549 alveolar epithelial cells with clinical CF isolates $(n=8)$, including NTHi50 and seven other CF clinical strains (supplementary table S2) and evaluated cytotoxicity by analysing cell viability $6 \mathrm{~h}$ after infection. We observed a similar cytotoxic potential of NTHi clinical strains. As shown in supplementary figure S1, only NTHi32, NTHi33 and NTHi34 isolates induced a modest (although statistically significant, $\mathrm{p}<0.05$ ) increase in cell toxicity in comparison to the NTHi reference strain. Overall, our data highlight that NTHi persistence, rather than NTHi intrinsic virulence, fuels the inflammatory burden in the lungs promoting chronic inflammation characterised by the sustained recruitment of neutrophils and T-cells.

\section{Discussion}

The pathogenic potential of chronic NTHi infection in lung disease is still controversial [1, 15]. Our research question was whether chronic NTHi infection could be considered as a potential risk factor for lung disease progression by fuelling the inflammatory burden. Our results demonstrate that chronic NTHi infection is associated with a sustained inflammatory burden, as also indicated by respiratory sample analysis in a small cohort of CF patients. The development of a new murine model was instrumental in mirroring chronic NTHi infection as observed in clinical settings and, consequently, in overtaking the apparent limited NTHi virulence observed in in vitro experiments with bacterial variants. In particular, we found out that chronic NTHi infection is associated with sustained recruitment of neutrophils in the lung. This pro-inflammatory response seems to be mainly mediated by type 17 immunity and by the presence of BALT in the sub-mucosal lung tissue. 
a)

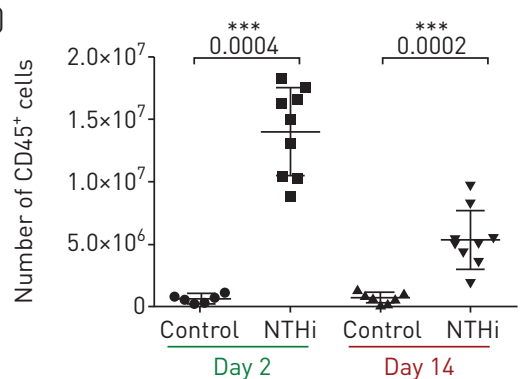

d)

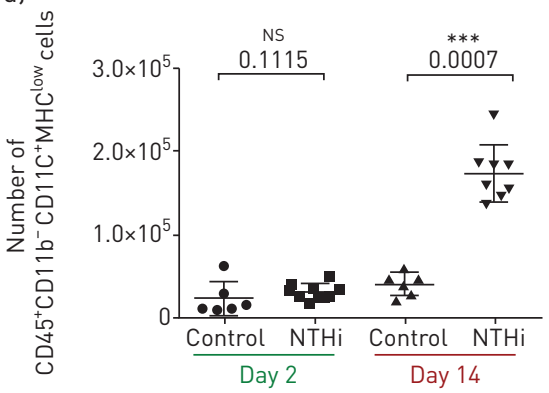

g)

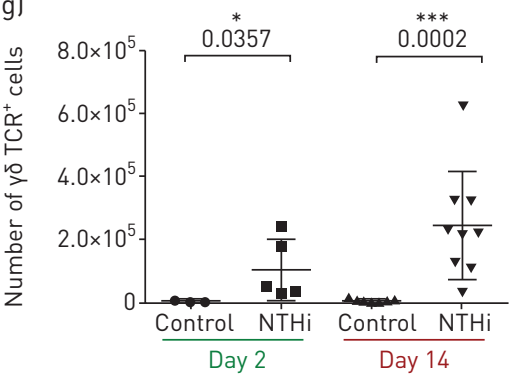

j)

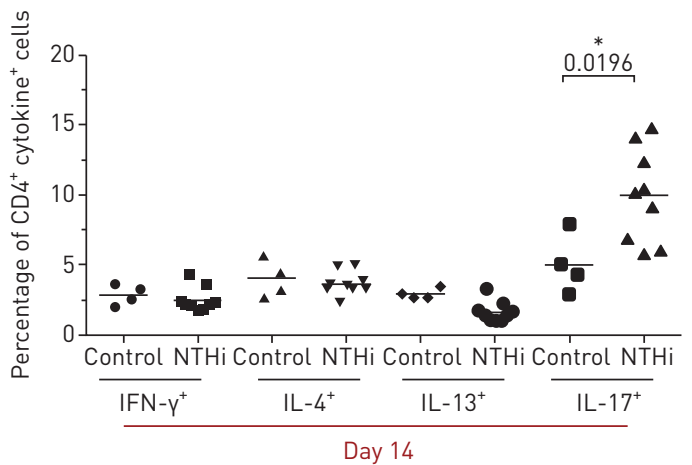

b)

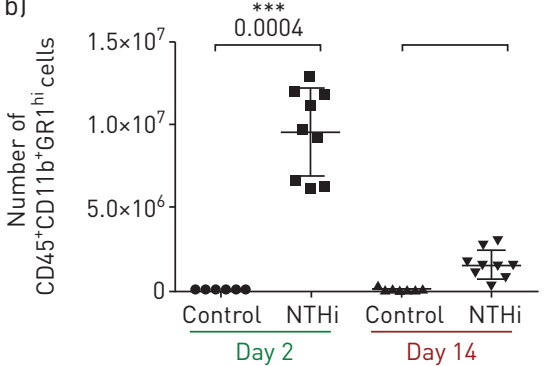

e)
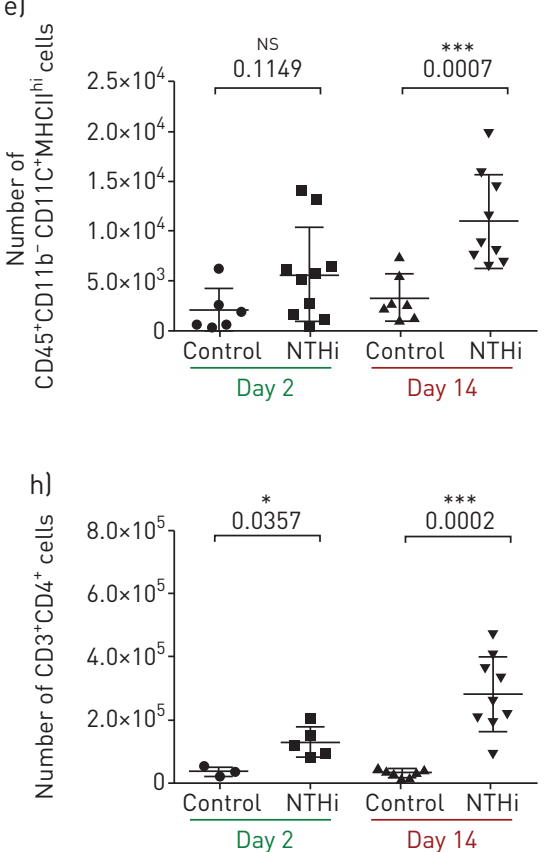

k)

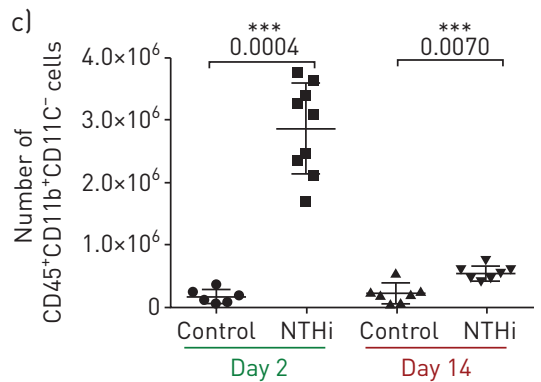

f)

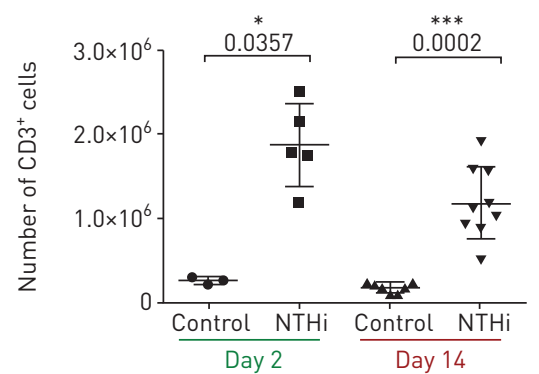

i)

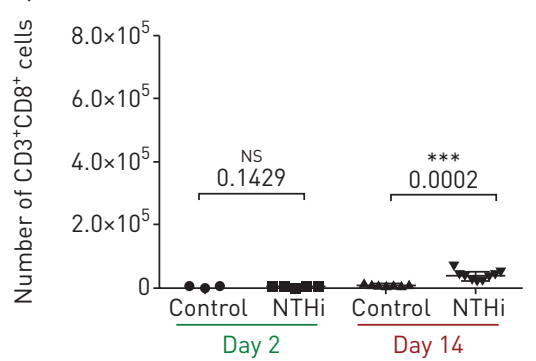

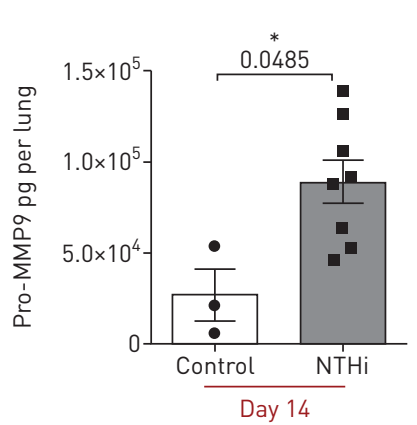

FIGURE 3 Immune response during the development of chronic nontypeable Haemophilus influenzae (NTHi) infection in mice. Mice were infected intratracheally with NTHi-embedded agar beads, and lungs were dissociated at 2 and 14 days post-infection. Flow cytometry was performed to quantify the total number of (a) leukocytes, (b) neutrophils, (c) monocytes/macrophages, (d) alveolar macrophages, (e) myeloid dendritic cells, (f) T-cells, (g) $\gamma \delta \mathrm{T}$-cells, (h) $\mathrm{CD}^{+} \mathrm{T}$-cells and (i) $\mathrm{CD}^{+}{ }^{+}$T-cells. Intracellular staining was performed ( $\mathrm{j}$ ) to identify interferon (IFN)- $\gamma$, interleukin (IL)-4, IL-13 or IL-17 producing CD4 $4^{+}$cells. (k) Pro-matrix metalloproteinase 9 (Pro-MMP9) was quantified by ELISA at 14 days post-infection. The data are pooled from at least two independent experiments $(n=3-9)$. ns: not significant; *: $p<0.05$; ${ }^{* *}$ : $p<0.01$; and ${ }^{* * *}$ : $p<0.001$ compared to control (Mann-Whitney $t$-test).

Epidemiological data on $H$. influenzae colonisation and disease risk were mainly collected from patients with non-CF bronchiectasis $[18,31,32]$. In the context of CF patients with NTHi colonisation/infection, only a few studies reported on associations with clinical characteristics, morbidity and mortality. $H$. influenzae is more frequently observed in CF patients without a positive history of $P$. aeruginosa lung 

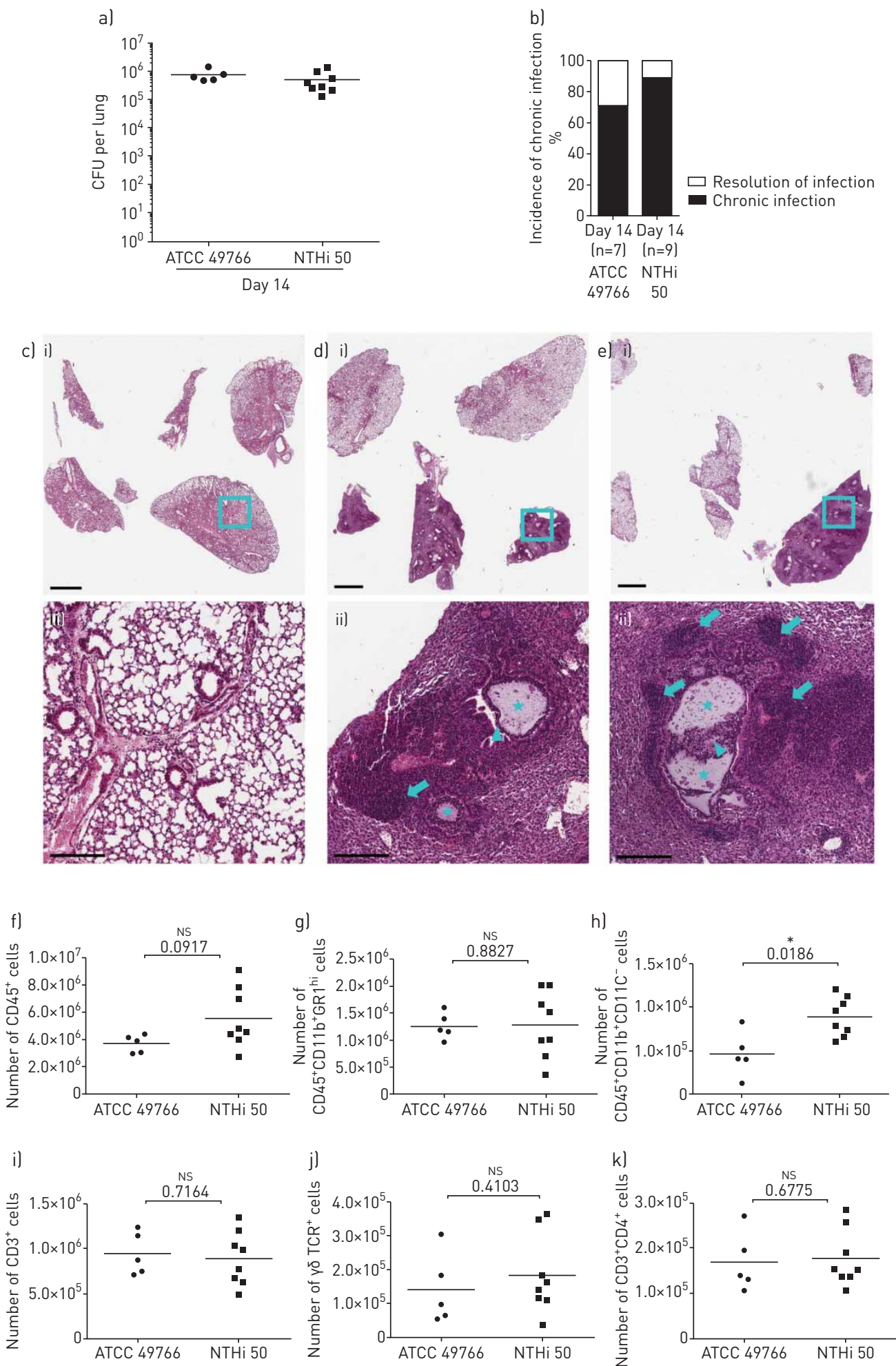

FIGURE 4 Bacterial burdens, histopathology and immune response in murine airways during chronic infection by clinical nontypeable Haemophilus influenzae (NTHi) strain. Mice were infected intratracheally with NTHi-embedded agar beads. Bacterial loads in the lung (a) and the incidence of chronic infection (b) were measured at 14 days post-infection. Haematoxylin and eosin staining of lung tissue sections was performed for control mice (c), ATCC 49766 (d) and NTHi 50 (e) infected mice. Stars indicate the presence of agar beads in bronchial lumens, arrowheads indicate the presence of neutrophils and macrophages in bronchial lumens, and arrows indicate the presence of lymphocyte aggregates. Representative images are shown from the i) lower (scale bar=2 $\mathrm{mm}$ ) to the ii) higher magnification (scale bar $=200 \mu \mathrm{m}$ ). Recruitment of (f) leukocytes, (g) neutrophils, (h) monocytes/ macrophages, (i) T-cells, (j) $\gamma \delta$ T-cells and ( $k$ ) $\mathrm{CD}^{+}{ }^{+} \mathrm{T}$-cells was measured by flow cytometric analysis in cell suspensions of murine lungs at 14 days post-infection. The data are pooled from at least two independent experiments $n=7$ (ATCC49766), $n=9$ (NTHi 50). NS: not significant; *: $p<0.05$ (Mann-Whitney $t$-test). 
Control

a)

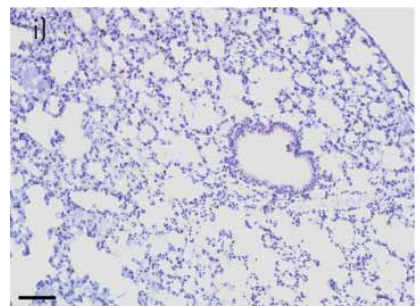

b)

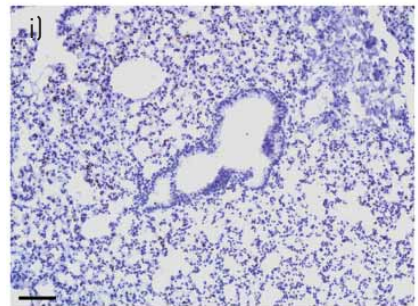

c)

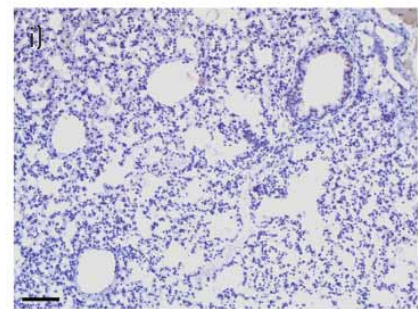

d)

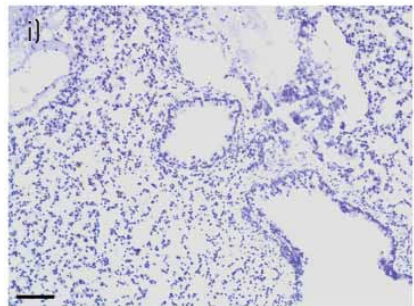

ATCC 49766
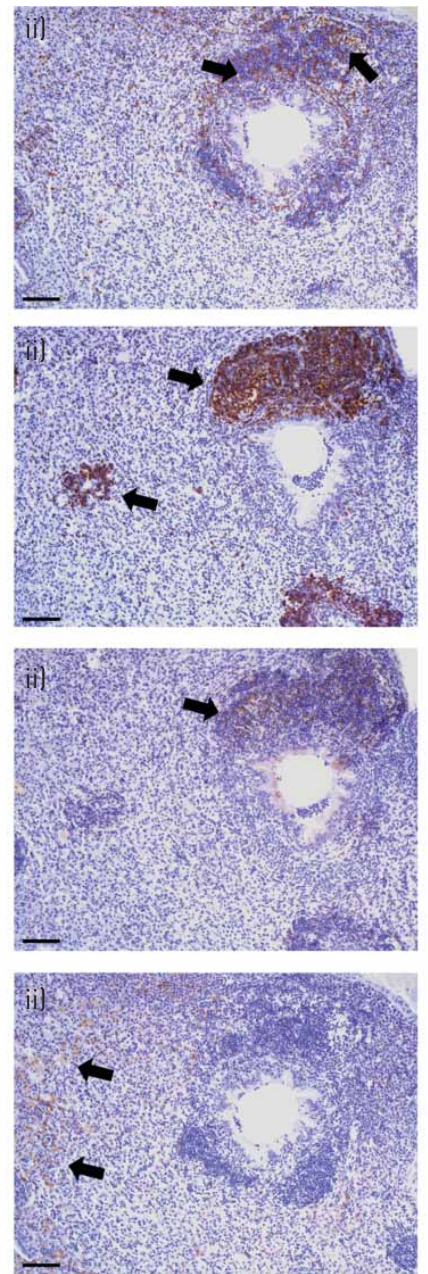

NTHi 50
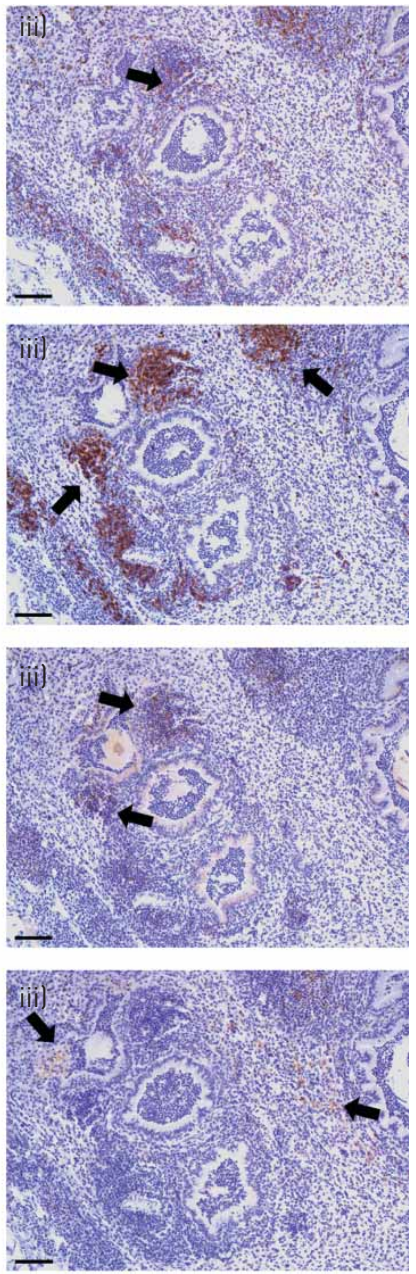

e)

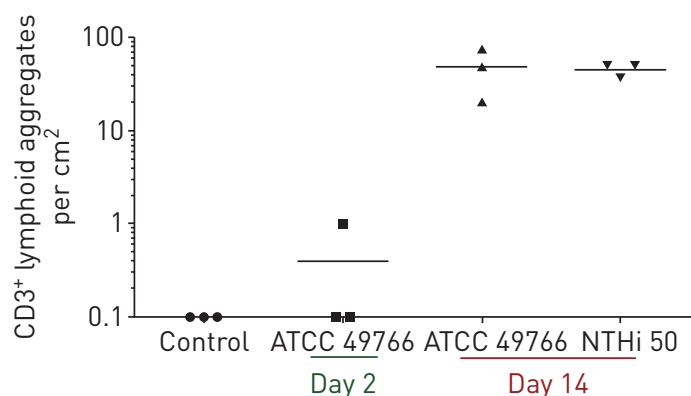

FIGURE 5 Chronic nontypeable Haemophilus influenzae (NTHi) infection promotes the formation of peribronchial lymphoid tissue structures. Mice were infected intratracheally with NTHi-embedded agar beads, and lung immunohistochemistry for the CD3 (a), B220 (b), CXCL13 (c), and F4/80 (d) molecules was performed on lung tissue sections at 14 days post-infection for control mice (i), ATCC 49766 (ii) and NTHi 50 (iii) infected mice (scale bar $=80 \mu \mathrm{m}$ ). (e) Morphometric analysis of $\mathrm{CD}^{+}$aggregates in murine lungs. Arrows indicate the presence of peribronchial lymphoid tissue structures. Horizontal bars represent median values. The data are pooled from at least two independent experiments (three mice/group).

infection, rather than in those who had been previously infected at least once [33,34]. Our data indicate that chronic infection by NTHi may account for a higher pro-inflammatory burden.

Interestingly, $H$. influenzae detection on respiratory tract culture was identified as a risk factor for substantial annual forced expiratory volume in $1 \mathrm{~s}\left(\mathrm{FEV}_{1}\right)$ decline in 4680 adolescents and young adults with CF from USA and Canada within the Epidemiologic Study of CF [35]. Although we recruited 
clinically stable CF patients with similar clinical conditions in term of $\mathrm{FEV}_{1}$, a limitation of our human results, due to the limited number of patients, is represented by the missing evaluation of the contribution of other confounding factors, such as genotype, age or sex. To further support our observations in CF patients, prospective observational clinical studies with a higher number of patients are required, possibly by setting up a multicenter study to increase availability of subjects with comparable clinical and demographic characteristics. In this context, the development of biological clinical sample banks (e.g. blood, respiratory samples or clinical strains) associated with a large clinical CF database may be useful to definitively address the potential of early NTHi infection as a risk factor for lung disease progression in CF.

As for clinical and basic science studies, the NTHi literature fails to deeply and fully discriminate colonisation from acute/intermittent or chronic infection/colonisation episodes of infection. The novelty of our work also relies on the dissection of the pro-inflammatory potential during chronic NTHi infection in the airways of mice. To date, animal models have been developed to mimic the acute phase of NTHi infection rather than the chronic phase, limiting steps forward in the field. Airway chronic infection by NTHi is not only common in CF disease and COPD, but also in other lung diseases with different aetiology, such as lung cancer [36-38]. In this context, CroAsdell et al. [39] exploited an acute infection model in which different amounts of NTHi were inoculated by oropharyngeal aspiration in C57BL/6J mice (up to $10^{8} \mathrm{CFU}$ ) and found that $24 \mathrm{~h}$ post-infection $>90 \%$ of bacteria had been cleared, independently from the inoculum dose. To date, mouse models based on the injection of NTHi planktonic bacterial cells in immunocompetent mice are useful for studying the acute NTHi infection phase rather than the chronic one.

The new mouse model developed in this study may reproduce the vicious cycles of infection, lung inflammation and tissue damage as observed in chronic respiratory disease mediated by NTHi infections. In the present study, we were able to evaluate the pro-inflammatory burden caused by chronic NTHi infection, associated with constitutive recruitment of neutrophils, as well as with increased levels of both $\gamma \delta^{+}$and $\mathrm{CD}^{+}$T-cell subsets in the advanced phase of lung infection. Previous studies identified a protective role of type 17 immunity (intended as IL-17-secreting cells) during transient and acute NTHi infection in mice $[23,36,40]$. Here, we found that chronic inflammation due to NTHi is associated with $\mathrm{CD}^{+} \mathrm{CD}^{+} \mathrm{IL}_{-1} 7^{+}$cells. As previously demonstrated for $P$. aeruginosa, we speculate that type 17 immunity can be a double-edged sword during NTHi infection: it could be protective by increasing host resistance in the early phase of infection, while being detrimental during the advanced phase of chronic infection by increasing the immunopathology $[11,14]$. Further studies using transgenic mice or inhibitory drugs could address this hypothesis.

Our data suggest that chronic NTHi infection in mice can induce an inflammatory response similar to those mediated by other well-known opportunistic pathogens (e.g. P. aeruginosa or Staphylococcus aureus) associated with chronic respiratory diseases. Here the NTHi-driven peribronchial lymphoid tissue structures, associated with sustained neutrophil recruitment, mimics pathological features similar to those observed for long-term S. aureus or P. aeruginosa infection in mice or humans with advanced diseases [14, 28]. In this context, the question whether the NTHi-inflammatory response promotes the infection of the airways by other pathogens such as P. aeruginosa or S. aureus, as occurs in CF patients, remains to be addressed.

Interestingly, our results with laboratory and clinical strains in mice suggest that the pro-inflammatory burden is mainly mediated by the immunopathological responses to chronic NTHi infection rather than by the specific pathogenic virulence of different bacterial strains. The in vitro results, obtained when different clinical isolates were tested, further support a similar pathogenicity. Of course, a study considering a higher number of clinical isolates is required to determine whether specific phenotypic traits can affect the pathogenic potential of different NTHi variants.

It is desirable that future studies would not exclusively aim at contrasting NTHi bacterial growth (e.g. with antimicrobial compounds) but would also target the NTHi-induced immunopathology (e.g. with anti-inflammatory drugs) in chronic lung disease. These approaches will help to better explore the contribution of long-term NTHi infection to disease progression, pulmonary exacerbations or super-infection events, such as the "predisposition" to P. aeruginosa infection. As already mentioned, NTHi infections are commonly associated with several chronic respiratory diseases with different aetiology $[3,4,18]$. Therefore, the murine model developed in this study will be exploitable to determine the contribution of NTHi chronic infections and new therapeutic opportunity in a broader spectrum of chronic lung diseases.

In conclusion, our results demonstrate that chronic NTHi infection, common during early lung disease, contributes to the exaggerated lung inflammatory burden and may contribute to declining lung function.

Acknowledgements: The authors thank A. Fiocchi (OMC, Mouse Histopathology Unit, San Raffaele Scientific Institute, Milan, Italy) for the mouse histopathology preparations and the San Raffaele Microscopy facility (Alembic) for images acquisition. 
Support statement: This work has been supported by an OSR_Seed Grant and Fondazione Cariplo (grant number 2016-0572) to N.I. Lorè. The funders had no role in study design, data collection and analysis, decision to publish, or preparation of the manuscript.

Author contributions: Conceiving and designing the experiments: N.I. Lorè, C. Cigana; performing experiments: F. Saliu, G. Rizzo, D. Girelli, S. Rizzetto, B. Sipione and N.I. Lorè; analysing data: F. Saliu, G. Rizzo, L. Cariani, V. Daccò, B. Sipione, C. Cigana and N.I. Lorè; performing statistical analysis: F. Saliu, G. Rizzo, V. Daccò and N.I. Lorè; interpretation of the experiment results: F. Saliu, G. Rizzo, A. Bragonzi, L. Cariani, D.M. Cirillo, C. Colombio, V. Daccò, C. Cigana and N.I. Lorè; preparing figures: F. Saliu, G. Rizzo and N.I. Lorè; writing the manuscript: F. Saliu, G. Rizzo, A. Bragonzi, L. Cariani, D.M. Cirillo, V. Daccò, C. Cigana and N.I. Lorè

Conflict of interest: F. Saliu has nothing to disclose. G. Rizzo has nothing to disclose. A. Bragonzi has nothing to disclose. L. Cariani has nothing to disclose. D.M. Cirillo has nothing to disclose. C. Colombo has nothing to disclose. V. Daccò has nothing to disclose. D. Girelli has nothing to disclose. S. Rizzetto has nothing to disclose. B. Sipione has nothing to disclose. C. Cigana has nothing to disclose. N.I. Lorè has nothing to disclose.

\section{References}

1 van Eldere J, Slack MPE, Ladhani S, et al. Non-typeable Haemophilus influenzae, an under-recognised pathogen. Lancet Infect Dis 2014; 14: 1281-1292.

2 Bakaletz LO, Novotny LA. Nontypeable Haemophilus influenzae (NTHi). Trends Microbiol 2018; 26: 727-728.

3 Su YC, Jalalvand F, Thegerström J, et al. The interplay between immune response and bacterial infection in COPD: focus upon non-typeable Haemophilus influenzae. Front Immunol 2018; 9: 1-26.

4 Sethi S, Murphy TF. Infection in the pathogenesis and course of chronic obstructive pulmonary disease. N Engl J Med 2008; 359: 2355-2365.

5 Sethi S. Infection as a comorbidity of COPD. Eur Respir J 2010; 35: 1209-1215.

6 Hartl D, Gaggar A, Bruscia E, et al. Innate immunity in cystic fibrosis lung disease. J Cyst Fibros 2012; 11: 363-382.

7 Shaker SB, von Wachenfeldt KA, Larsson S, et al. Identification of patients with chronic obstructive pulmonary disease (COPD) by measurement of plasma biomarkers. Clin Respir J 2008; 2: 17-25.

8 Tümmler B. Progress in understanding the molecular pathology and microbiology of cystic fibrosis. Lancet Respir Med 2020; 8: 8-10.

9 Angelis N, Porpodis K, Zarogoulidis P, et al. Airway inflammation in chronic obstructive pulmonary disease. J Thorac Dis 2014; 6: 4-9.

10 Roesch EA, Nichols DP, Chmiel JF. Inflammation in cystic fibrosis: an update. Pediatr Pulmonol 2018; 53 S30-S50.

11 Lorè NI, Bragonzi A, Cigana C. The IL-17A/IL-17RA axis in pulmonary defence and immunopathology. Cytokine Growth Factor Rev 2016; 30: 19-27.

12 Malhotra S, Hayes D, Jr, Wozniak DJ. Pseudomonas aeruginosa: the host-microbe interface. Clin Microbiol Rev 2019; 32: 1-46.

13 Cigana C, Lorè NI, Riva C, et al. Tracking the immunopathological response to Pseudomonas aeruginosa during respiratory infections. Sci Rep 2016; 6: 1-12.

14 Lorè NI, Cigana C, Riva C, et al. IL-17A impairs host tolerance during airway chronic infection by Pseudomonas aeruginosa. Sci Rep 2016; 6: 25937.

15 Hiller EJ, Ispahani P, Baker M. Haemophilus infection. Arch Dis Child 1990; 65: 255-258.

16 Wang Z, Maschera B, Lea S, et al. Airway host-microbiome interactions in chronic obstructive pulmonary disease. Respir Res 2019; 8: 1-14.

17 Dicker AJ, Huang JTJ, Lonergan M, et al. The sputum microbiome, airway inflammation, and mortality in chronic obstructive pulmonary disease. J Allergy Clin Immunol 2021; 147: 158-167.

18 Tufvesson E, Markstad H, Bozovic G, et al. Inflammation and chronic colonization of Haemophilus influenzae in sputum in COPD patients related to the degree of emphysema and bronchiectasis in high-resolution computed tomography. Int J Chron Obstruct Pulmon Dis 2017; 12: 3211-3219.

19 Tufvesson E, Bjermer L, Ekberg M. Patients with chronic obstructive pulmonary disease and chronically colonized with Haemophilus influenzae during stable disease phase have increased airway inflammation. Int J Chron Obstruct Pulmon Dis 2015; 10: 881-889.

20 Cardines R, Giufrè M, Pompilio A, et al. Haemophilus influenzae in children with cystic fibrosis: antimicrobial susceptibility, molecular epidemiology, distribution of adhesins and biofilm formation. Int J Med Microbiol 2012 302: $45-52$.

21 Aziz A, Sarovich DS, Nosworthy E, et al. Molecular signatures of nontypeable Haemophilus influenzae lung adaptation in pediatric chronic lung disease. Front Microbiol 2019; 10: 1622

22 Garmendia J, Viadas C, Calatayud L, et al. Characterization of nontypable Haemophilus influenzae isolates recovered from adult patients with underlying chronic lung disease reveals genotypic and phenotypic traits associated with persistent infection. PLoS One 2014; 9: e97020.

23 Roos AB, Sethi S, Nikota J, et al. IL-17A and the promotion of neutrophilia in acute exacerbation of chronic obstructive pulmonary disease. Am J Respir Crit Care Med 2015; 192: 428-437.

24 Spagnuolo L, de Simone M, Lorè NI, et al. The host genetic background defines diverse immune-reactivity and susceptibility to chronic Pseudomonas aeruginosa respiratory infection. Sci Rep 2016; 6: 36924.

25 Kriegeskorte A, Lorè I, Bragonzi A, et al. Thymidine-dependent Staphylococcus aureus small-colony variant are induced by trimethoprim-sulfamethoxazole (SXT) and have increased fitness during SXT challenge. Antimicrob Agents Chemother 2015; 59: 7265-7272.

26 Price AE, Reinhardt RL, Liang HE, et al. Marking and quantifying IL-17A-producing cells in vivo. PLoS One 2012; 7: e39750.

27 Drusian L, Nigro EA, Mannella V, et al. mTORC1 upregulation leads to accumulation of the oncometabolite fumarate in a mouse model of renal cell carcinoma. Cell Rep 2018; 24: 1093-1104.e6. 
Frija-Masson J, Martin C, Regard L, et al. Bacteria-driven peribronchial lymphoid neogenesis in bronchiectasis and cystic fibrosis. Eur Respir J 2017; 49: 1601873.

29 Singer M, Sansonetti PJ. IL-8 is a key chemokine regulating neutrophil recruitment in a new mouse model of Shigella-induced colitis. J Immunol 2004; 173: 4197-4206.

30 Greenlee KJ, Werb Z, Kheradmand F. Matrix metalloproteinases in lung: multiple, multifarious, and multifaceted. Physiol Rev 2007; 87: 69-98.

31 Goeminne PC, Scheers H, Decraene A, et al. Risk factors for morbidity and death in non-cystic fibrosis bronchiectasis: a retrospective cross-sectional analysis of CT diagnosed bronchiectatic patients. Respir Res 2012; 13: $1-8$.

32 Richardson H, Dicker AJ, Barclay H, et al. The microbiome in bronchiectasis. Eur Respir Rev 2019; $28: 190048$.

33 Vongthilath R, Thiriez BR, Dehillotte C, et al. Clinical and microbiological characteristics of cystic fibrosis adults never colonized by Pseudomonas aeruginosa: analysis of the French CF registry. PLoS One 2019; 14: 1-9.

34 Hector A, Kirn T, Ralhan A, et al. Microbial colonization and lung function in adolescents with cystic fibrosis. J Cyst Fibros 2016; 15: 340-349.

35 Vandenbranden SL, McMullen A, Schechter MS, et al. Lung function decline from adolescence to young adulthood in cystic fibrosis. Pediatr Pulmonol 2012; 47: 135-143.

36 Li W, Zhang X, Yang Y, et al. Recognition of conserved antigens by Th17 cells provides broad protection against pulmonary Haemophilus influenzae infection. Proc Natl Acad Sci 2018; 115: 201802261. www.pnas.org/lookup/ doi/10.1073/pnas.1802261115.

37 Euba B, López-López N, Rodríguez-Arce I, et al. Resveratrol therapeutics combines both antimicrobial and immunomodulatory properties against respiratory infection by nontypeable Haemophilus influenzae. Sci Rep 2017; 7: $1-14$.

38 Chang SH, Mirabolfathinejad SG, Katta H, et al. T helper 17 cells play a critical pathogenic role in lung cancer Proc Natl Acad Sci U S A 2014; 111: 5664-5669.

39 Croasdell A, Lacy SH, Thatcher TH, et al. Resolvin D1 dampens pulmonary inflammation and promotes clearance of nontypeable Haemophilus influenzae. J Immunol 2016; 196: 2742-2752. www.jimmunol.org/lookup/doi/10. 4049/jimmunol.1502331.

40 Noda K, Kodama S, Umemoto S, et al. Th17 cells contribute to nontypeable Haemophilus influenzae-specific protective immunity induced by nasal vaccination with P6 outer membrane protein and $\alpha$-galactosylceramide. Microbiol Immunol 2011; 55: 574-581. 